\title{
Atypical central retinal artery occlusion as the first presentation of POEMS syndrome: a case report
}

\author{
Panitha Jindahra', Charungthai Dejthevaporn ${ }^{1}$, Pimjai Niparuck', Jariya Waisayarat ${ }^{2}$, Piyaphon Cheecharoen ${ }^{3}$, \\ Thanatporn Threetong ${ }^{4}$, Purit Petpiroon ${ }^{4}$, Tharikarn Sujirakul ${ }^{4}$, Anuchit Poonyathalang ${ }^{4}$ and Kavin Vanikieti ${ }^{4 *}$
}

\begin{abstract}
Background: POEMS syndrome is a plasma cell disorder, which clinically manifests from paraneoplastic syndrome: polyneuropathy, organomegaly, endocrinopathy, monoclonal plasma cell disorder, and skin changes. The most common ocular manifestation is optic disc swelling, whereas other ocular manifestations; cystoid macular edema, serous macular detachment, venous sinus thrombosis, infiltrative orbitopathy, uveitis, neovascularization of the disc, peripapillary choroidal neovascularization and optic disc drusen, had also been reported.

Case presentation: A 52-year-old Thai man presented with 5-day sudden painless visual loss in the left eye. Ocular examination revealed visual acuity of 20/20 and no light perception in the right and left eye, respectively. Right fundoscopic examination was significant for hyperemic generalized optic disc swelling. Left fundoscopic examination revealed opaque and edematous entire retina giving the appearance of central retinal artery occlusion (CRAO) along with pallid "chalky white" optic disc swelling. Fluorescein angiography showed profound leakage of bilateral optic nerve heads and arteriolar filling defect in macular area along with leakage of small retinal arterioles in the left eye. Indocyanine green angiography demonstrated choroidal filling defect in the left eye only. Neuroimaging showed enhancement and luminal narrowing of left internal carotid artery, early subacute watershed infarctions in the left cerebral hemisphere and pachymeningeal enhancement. Cerebrospinal fluid analysis revealed high protein level with normal opening pressure. Intravenous methylprednisolone was initially started without any benefit. After extensive investigations, diagnosis of "POEMS syndrome" was made based on polyneuropathy, elevated lambda light chain level, elevated plasma vascular endothelial growth factor (VEGF), hepatomegaly, spinal sclerotic bone lesions, and thrombocytosis. Furthermore, sural nerve biopsy demonstrated neuropathy and positive VEGF staining. He was treated with eight cycles of bortezomib, cyclophosphamide and dexamethasone (BorCyDex). Polyneuropathy and thrombocytosis had remarkably improved after 2nd cycle, whereas, visual impairment had shown no recovery. Hepatomegaly was significantly reduced after the completion of BorCyDex. Our case eventually received autologous hematopoietic stem cell transplantation with high dose melphalan.
\end{abstract}

Conclusions: To our knowledge, we illustrated the first patient given CRAO as the first presentation and ocular finding ever reported in POEMS syndrome. Both cerebral and ocular infarctions were presumably the result of VEGF-induced cranial vasculopathy as evidenced by neuroimaging.

Keywords: POEMS syndrome, Central retinal artery occlusion, Anterior ischemic optic neuropathy, Visual loss

\footnotetext{
* Correspondence: vanikieti.kavin@gmail.com

${ }^{4}$ Department of Ophthalmology, Faculty of Medicine Ramathibodi Hospital,

Mahidol University, 270 Rama VI Road, Bangkok 10400, Thailand

Full list of author information is available at the end of the article
}

(c) The Author(s). 2018 Open Access This article is distributed under the terms of the Creative Commons Attribution 4.0 International License (http://creativecommons.org/licenses/by/4.0/), which permits unrestricted use, distribution, and reproduction in any medium, provided you give appropriate credit to the original author(s) and the source, provide a link to the Creative Commons license, and indicate if changes were made. The Creative Commons Public Domain Dedication waiver (http://creativecommons.org/publicdomain/zero/1.0/) applies to the data made available in this article, unless otherwise stated. 


\section{Background}

POEMS syndrome is a plasma cell disorder, which clinically manifests from paraneoplastic syndrome: polyneuropathy, organomegaly, endocrinopathy, monoclonal plasma cell disorder, and skin changes [1]. The most common ocular manifestation is optic disc swelling, whereas other ocular manifestations; cystoid macular edema (CME), serous macular detachment, venous sinus thrombosis, infiltrative orbitopathy, uveitis, neovascularization of the disc (NVD), peripapillary choroidal neovascularization and optic disc drusen, had also been reported [2-12]. To our knowledge, central retinal artery occlusion has never been reported in POEMS syndrome. We herein describe the first reported patient with POEMS syndrome who initially presented with atypical central retinal artery occlusion.

\section{Case presentations}

A 52-year-old Thai man presented with sudden painless visual loss in his left eye upon awakening 5 days prior to presentation. He had no visual complaints regarding his right eye. He reported no fever, jaw claudication, general malaise, anorexia, hearing of a whooshing sound, or new-onset headache. His ocular history was significant for bilateral hyperopia. His medical history was notable for $18.5-\mathrm{kg}$ body weight loss in the past 2 years. He was not taking any medications and was a nonsmoker.

Ocular examination revealed visual acuity of 20/20 and no light perception in the right eye and left eye, respectively. The presence of a grade $4+$ relative afferent pupillary defect of the left eye was noted. The intraocular pressure was $15 \mathrm{mmHg}$ bilaterally. Slit-lamp examination showed a mild cortical cataract without signs of ocular inflammation bilaterally. Fundoscopic examination of the left eye showed that the entire retina was opaque and edematous, particularly in the posterior pole, and pallid "chalky white" generalized optic disc swelling was noted (Fig. 1b). Neither emboli nor cottonwool spots were detected. Fundoscopic examination of the right eye was significant only for hyperemic generalized optic disc swelling; no hemorrhage, cotton-wool spots, or exudates were present (Fig. 1a).

A color vision test (Lanthony desaturated 15-hue test) of the right eye was unremarkable. The Humphrey 24-2 automated static perimetry program of the right eye showed blind spot enlargement. The average retinal nerve fiber layer thickness of the optic nerve head measured by

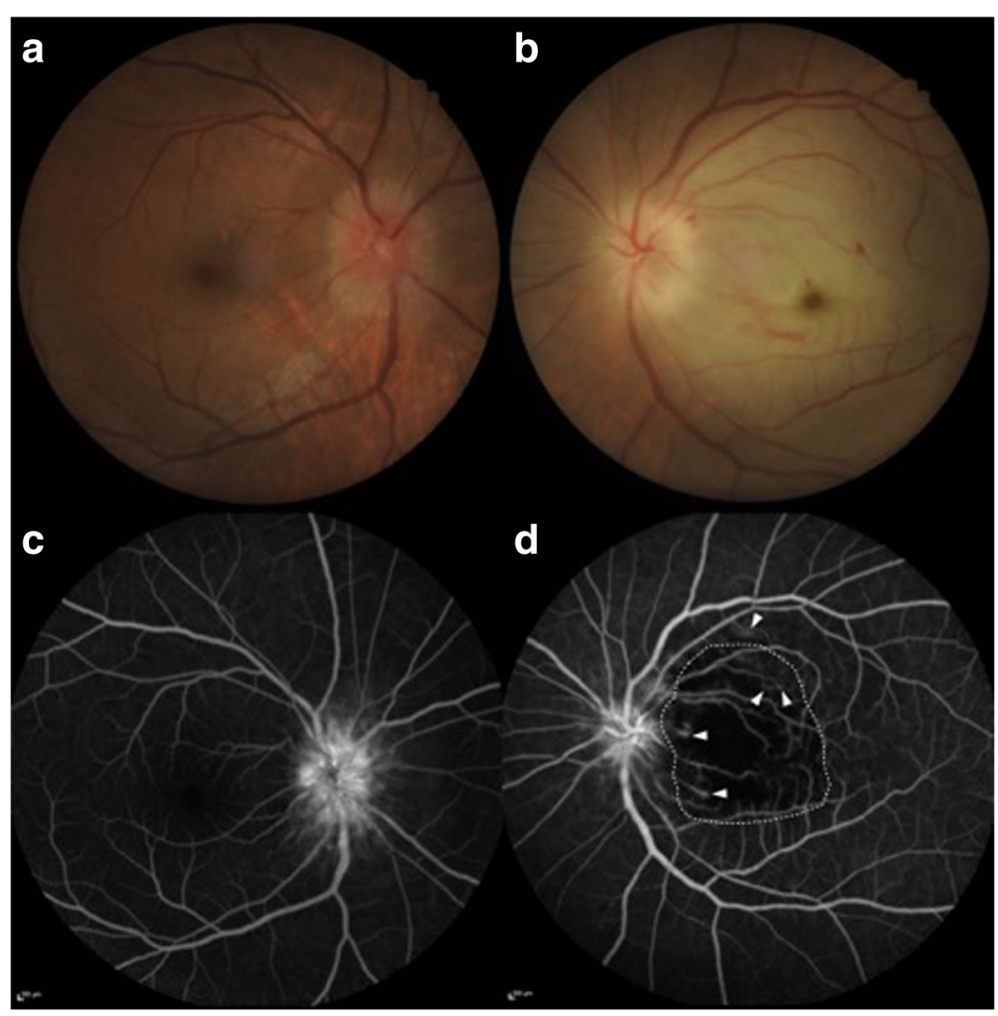

Fig. 1 Fundus photograph and fundus fluorescein angiography. a Hyperemic and generalized optic disc swelling is observed in the right eye. $\mathbf{b}$ An opaque and edematous retina in the posterior pole along with pallid "chalky white" generalized optic disc swelling is demonstrated in the left eye. c Fundus fluorescein angiography showing profound leakage of the optic nerve head in the right eye. $\mathbf{d}$ Leakage of the optic nerve head, filling defects in multiple arterioles predominantly in the macular area (area within the dashed lines), and leakage of small retinal arterioles (arrowheads) are observed in the left eye 
spectral-domain optical coherence tomography (Cirrus HD-OCT, software version 6.0.0.599; Carl Zeiss Meditec, Inc., Dublin, CA, USA) was 554 and $187 \mu \mathrm{m}$ in the right and left eye, respectively. Fundus fluorescein angiography (FFA) revealed profound leakage of the bilateral optic nerve heads, and filling defects were observed in multiple arterioles, predominantly in the macular area of the left eye, along with leakage of small retinal arterioles in the left eye (Fig. 1c, d). No filling defect or retinal vessel leakage was noted in the right eye. Indocyanine green angiography (ICG) demonstrated a choroidal filling defect in the left eye only. Partial occlusion of both retinal and choroidal circulation in the left eye was suspected. An electroretinogram (ERG) was evaluated according to the International Society for Clinical Electrophysiology of Vision standard (MonColor; Metrovision, Perenchies, France). A full-field ERG of the left eye showed a significant reduction of both the awave and b-wave amplitude, along with a decreased b:a ratio with a normal implicit time in the photopic and maximal combined responses, confirming both retinal and choroidal circulation in the left eye was affected. An ERG of the right eye was normal. Both sides of the superficial temporal artery were pulsatile and painless. The patient's blood pressure was $130 / 80 \mathrm{mmHg}$. A provisional diagnosis of atypical central retinal artery occlusion (CRAO) with anterior ischemic optic neuropathy (AION) in the left eye in conjunction with generalized right optic disc swelling was made. Treatment was started with intravenous methylprednisolone at $1 \mathrm{~g}$ once daily for 5 days.

A complete blood count showed $5.7 \times 10^{9} / \mathrm{L}$ white blood cells with $76 \%$ neutrophils; the platelet count was $595 \times 10^{9}$ / $\mathrm{L}$, and the hemoglobin level was $17.3 \mathrm{~g} / \mathrm{dL}$. The erythrocyte sedimentation rate was $9 \mathrm{~mm} / \mathrm{h}$, and the C-reactive protein level was $1.14 \mathrm{mg} / \mathrm{L}$. The results of a comprehensive vasculitis panel, including measurement of antinuclear antibody, cytoplasmic antineutrophil cytoplasmic antibodies, perinuclear antineutrophil cytoplasmic antibodies, and antidouble-stranded DNA, were within normal limits. The results of the serum Venereal Disease Research Laboratory (VDRL) test, Treponema pallidum hemagglutination (TPHA) test, and anti-human immunodeficiency virus antibody test were negative. A metabolic panel, including liver function tests, the fasting blood sugar level, and a lipid profile, was unremarkable. Antiphospholipid antibodies were negative. The results of a coagulation panel were within normal limits.

Magnetic resonance imaging (MRI) of the brain with gadolinium injection revealed early subacute left anterior cerebral artery/middle cerebral artery watershed infarction, early subacute left middle cerebral artery/posterior cerebral artery watershed infarction, and marked pachymeningeal thickening with enhancement of the bilateral cerebral convexities (Fig. 2d). Diffusion weighted imaging (DWI) of the brain showed restricted diffusion involving gray-white matter of the left parietal lobe and centrum semiovale. Moreover, enhancement and luminal narrowing of the petrous and supraclinoid left internal carotid artery (ICA) were observed (Fig. 2a-c). The bilateral optic nerves showed a normal size and signal intensity. Magnetic resonance angiography (MRA) revealed luminal narrowing from the distal cervical left ICA extending to the supraclinoid left ICA. The patient was treated with aspirin. Echocardiography and electrocardiography showed normal results.

A lumbar puncture was then performed, and $6 \mathrm{~mL}$ of clear cerebrospinal fluid (CSF) was obtained with an opening pressure of $19 \mathrm{cmH}_{2} \mathrm{O}$. CSF analysis revealed a significantly high protein level $(234.6 \mathrm{mg} / \mathrm{dL})$, normal glucose concentration $(61 \mathrm{mg} / \mathrm{dL})$, and white cell count of 4 cells/ $\mathrm{mm}^{3}$ (100\% mononuclear cells). The VDRL test, TPHA test, polymerase chain reaction (PCR), and cultures for microorganisms were all negative in the CSF.

No clinical improvement was demonstrated during the 5-day course of methylprednisolone, and patient developed numbness in both feet.

Serum protein electrophoresis revealed no hypergammaglobulinemia, whereas, an IgG $\lambda$ monoclonal gammopathy was detected by serum immunofixation electrophoresis (Fig. 3a). In addition, computed tomography (CT) of the whole spine showed multiple mixed lytic osteosclerotic bone lesions involving the T8 and L1 bodies (Fig. 3b), bilateral 8th ribs, right ilium, and right sacrum.

A more comprehensive systemic examination showed a symmetrical decrease in the motor power of the bilateral extensor hallucis longus and a symmetrical decrease in sensation (including proprioception, vibration, pain, and temperature) of both feet. The Achilles and patellar reflexes were absent bilaterally. A nerve conduction study also revealed sensory and motor demyelinating polyneuropathy. The Romberg sign was positive and steppage gait was noted. Moreover, hepatomegaly was observed on a CT scan of the whole abdomen, and localized hypertrichosis associated with hyperpigmentation involving the dorsal aspect of both feet was noted (Fig. 3c).

A diagnosis of POEMS syndrome was made. The levels of hormones, plasma interleukin-6, and plasma vascular endothelial growth factor (VEGF) were measured and PCR for human herpesvirus type 8 in serum was performed to support this diagnosis. The interleukin- 6 level was $<1.5 \mathrm{pg} /$ $\mathrm{mL}$ (reference range, $0-7 \mathrm{pg} / \mathrm{mL}$ ), and the plasma VEGF level was $1828 \mathrm{pg} / \mathrm{mL}$ (reference range, $0-860 \mathrm{pg} / \mathrm{mL}$ ). PCR for human herpesvirus type 8 in serum was negative. The levels of hormones, including testosterone, free T3, free T4, thyroid-stimulating hormone, and cortisol, were normal.

A bone marrow biopsy demonstrated normocellular bone marrow; adequate red cells, white cells, and platelets; and scattered plasma cells. CD138 immunohistochemistry showed only $5 \%$ plasma cells. 


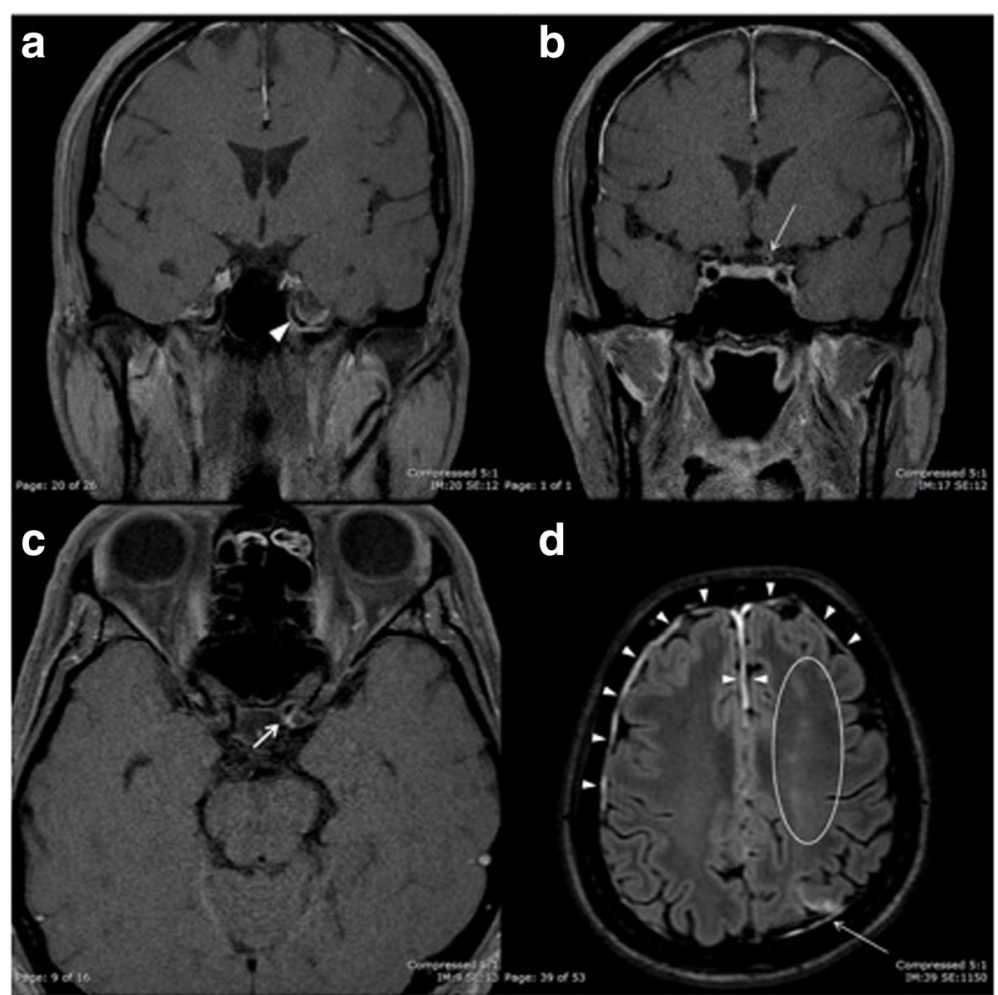

Fig. 2 Magnetic resonance imaging of the brain with gadolinium injection. $\mathbf{a}$, b Coronal T1-weighted images with gadolinium injection showing enhancement and luminal narrowing of the petrous (arrowhead) and supraclinoid (arrow) left internal carotid artery. c Axial T1-weighted image with gadolinium injection showing enhancement and luminal narrowing of the supraclinoid (arrow) left internal carotid artery. $\mathbf{d}$ Axial fluid attenuation inversion recovery (FLAIR) image with gadolinium injection showing early subacute left anterior cerebral artery/middle cerebral artery watershed infarction (area within the circle), early subacute left middle cerebral artery/posterior cerebral artery watershed infarction (arrow), and marked pachymeningeal thickening with enhancement of the bilateral cerebral convexities (arrowheads)

Sural nerve biopsy was also undertaken and demonstrated neuropathy through slightly decreased myelinated fibers density (Fig. 3d). Mild to moderately increased small vessels in epineurium led to the diagnosis of POEMS syndrome, which confirmed by a positive VEGF (Fig. 3e). There was no evidence of vasculitis or amyloidosis. The patient was treated with eight cycles of bortezomib, cyclophosphamide, and dexamethasone (BorCyDex). After the 2nd cycle of BorCyDex, the polyneuropathy had remarkably improved and the platelet count returned to normal; however, the visual loss in the left eye was still present. Disease assessment was performed after the 4th and 8th cycles of BorCyDex. CSF was obtained and showed high protein levels (200 and $150 \mathrm{mg} / \mathrm{dL}$ after the 4th and 8th cycle of treatment, respectively). Hepatomegaly was significantly reduced based on CT. After eight cycles of BorCyDex, the patient finally underwent autologous hematopoietic stem cell transplantation with high-dose melphalan.

\section{Discussion and conclusions}

Our patient was diagnosed with POEMS syndrome based on demyelinating polyneuropathy, lambda light chainrestricted monoclonal plasma cell proliferative disorder, sclerotic bone lesions, plasma VEGF elevation, hepatomegaly, hypertrichosis associated with hyperpigmentation, thrombocytosis, and bilateral optic disc swelling [1].

To our knowledge, this is the first reported patient with POEMS syndrome to exhibit CRAO as the first presentation. Optic disc swelling is the most common ocular finding and occurs in 52.0 to $67.5 \%$ of affected patients $[2,3]$.

Other ocular manifestations reported in the literature include cystoid macular edema [4-6], serous macular detachment [7, 8], venous sinus thrombosis [9], infiltrative orbitopathy [10], uveitis [11], neovascularization of the disc [10], peripapillary choroidal neovascularization, and optic disc drusen [12].

Our patient was considered to have atypical CRAO given the demonstration of small retinal arteriole leakage on FFA, association with AION, and contralateral generalized optic disc swelling.

Our hypothesis regarding the mechanism causing leakage of small retinal arterioles on FFA in the present case is microvascular hyperpermeability induced by an increased plasma VEGF level. VEGF is thought to be the major contributor to various manifestations of POEMS syndrome, including ocular findings $[1,2,6,13]$. 


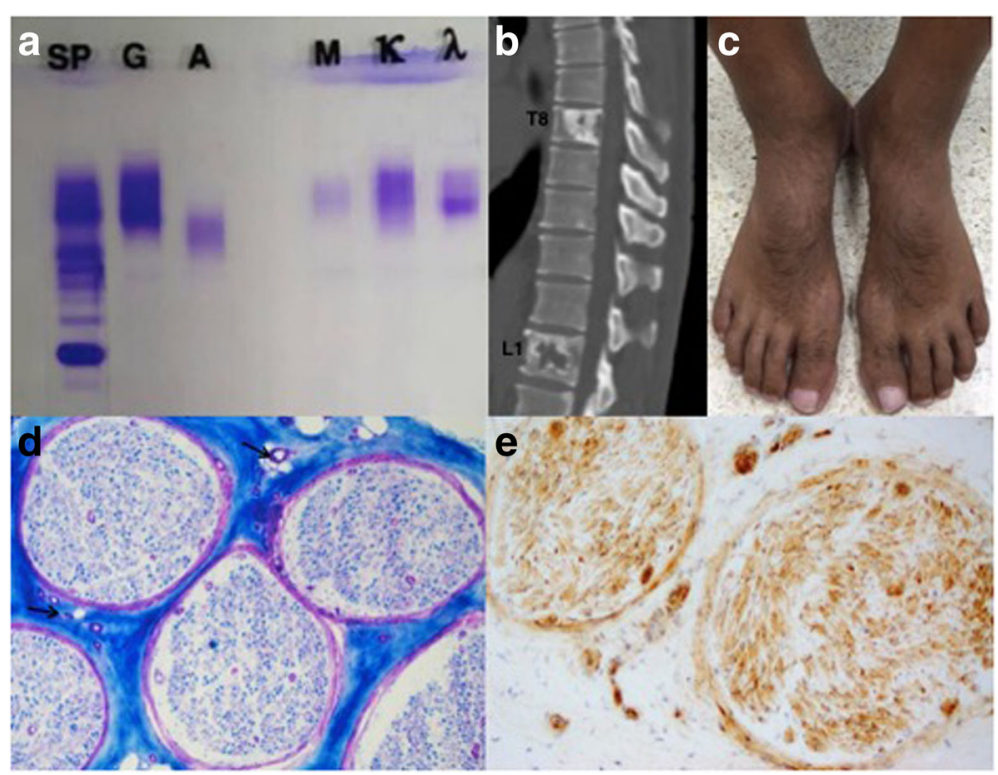

Fig. 3 Serum immunofixation electrophoresis, computed tomography of the spine, external appearance of both feet, and sural nerve biopsy. a Serum immunofixation electrophoresis showing an $\lg \mathrm{G} \lambda$ monoclonal gammopathy. $\mathbf{b}$ Computed tomography of the spine with contrast showing multiple mixed lytic osteosclerotic bone lesions involving the T8 and L1 bodies. c Localized hypertrichosis associated with hyperpigmentation involving the dorsal aspect of both feet. Sural nerve biopsy showing neuropathy through slightly decreased myelinated fibers density with mild to moderate increase of small vessels in epineurium (arrows) (d: Luxol Fast Blue staining, 100x) and positive VEGF staining (e: VEGF staining, 200x)

The association with AION was the second atypical characteristic of CRAO in this case. The typical pallid "chalky white" generalized optic disc swelling led us to highly suspect paraoptic short posterior ciliary artery involvement. Inability to perceive light was caused by acute ischemia of not only the retina but also the optic nerve, which is supplied by the choroidal circulation. This was confirmed by a full-field ERG, which showed a significant reduction in both the a-wave and b-wave amplitude. Moreover, indocyanine green angiography of the left eye revealed a choroidal filling defect, consistent with short posterior ciliary artery occlusion. Ophthalmic artery occlusion was the most likely explanation for the ischemia of both the retina and optic nerve. This was supported by the findings of luminal narrowing of the left ICA on MRI and MRA.

The last atypical characteristic of CRAO was the generalized optic disc swelling in the right eye. Given the wellpreserved visual function of the right eye despite the appearance of marked swelling of the optic disc, arteritic AION of the right eye was less of a concern. Considering the normal CSF opening pressure, the cause of the optic disc swelling in the right eye was probably microvascular hyperpermeability induced by the increased plasma VEGF level. This speculation is consistent with many previous reports $[1,2,6,13]$. However, the pathophysiology of optic disc swelling remains unclear.

Cerebral infarctions in patients with POEMS syndrome have been previously described [14-18]. The area of infarction varies and may include the end arteries, watershed areas, and cervical and proximal intracranial vasculature [14]. Potential etiologies remain inconclusive. Dupont et al. [14] reported that an elevated platelet count and evidence of plasma cell proliferation on bone marrow biopsy lead to an increased risk of ischemic stroke. These findings are consistent with the present case.

In our patient, vasculopathy of the left ICA based on MRI and MRA could explain both the cerebral infarctions and the CRAO associated with AION. Moreover, hyperviscosity secondary to thrombocytosis was thought to be the synergic risk factor that led to the arterial thrombosis events.

Arterial walls enhancement has rarely been reported. Fu et al. [18] described a patient with POEMS syndrome who developed multiple acute watershed infarctions of the left cerebral hemisphere associated with enhancement and luminal narrowing of the ipsilateral ICA. These findings were similar to those in our patient. Moreover, generalized cranial pachymeningeal involvement was observed in our patient. This is consistent with a previous study reported by Briani et al. [19], who described nine patients with POEMS syndrome who showed pachymeningeal thickening with intense enhancement. Furthermore, two of nine patients showed hyperplasia of meningothelial cells, neovascularization, noninflammatory obstructive vessel remodeling, and strong coexpression of VEGF and VEGF receptor on meningeal biopsy. These findings suggest that VEGF 
might be a major contributor in the pathogenesis of pachymeningeal involvement in POEMS syndrome. We speculate that the possible cause of arterial wall enhancement might be similar to that of VEGF-induced pachymeningeal enhancement. However, we did not perform a biopsy of any affected vasculature or meninges.

With respect to the vasculopathy of the left ICA based on MRI and MRA and leakage of the bilateral optic nerve heads and small retinal arterioles in the left eye on FFA, our patient demonstrated involvement of all sizes of affected arterial vessels ranging from the distal cervical ICA to small retinal arterioles. Elevation of the plasma VEGF level is proposed to be the major contributor to these abnormalities.

Sural nerve biopsy is recommended at baseline if clinically indicated [1]. Symmetrical decrease in sensation of both feet was exhibited in our patient. This was supported by a nerve conduction study, which revealed sensory demyelinating polyneuropathy. Similarly, a reduction in the myelinated fiber population was revealed pathologically. In addition, VEGF overexpression and VEGF-related proliferation of endothelial cells as evidenced by the biopsy supported the diagnosis of POEMS syndrome [1].

In conclusion, we have herein reported the first patient with CRAO as the initial presentation and ocular finding of POEMS syndrome. The mechanism of occlusion remains unclear due to its rarity. Further studies are needed to determine the definite pathophysiology.

\begin{abstract}
Abbreviations
AION: Anterior ischemic optic neuropathy; BorCyDex: Bortezomib, cyclophosphamide, and dexamethasone; CME: Cystoid macular edema; CRAO: Central retinal artery occlusion; CSF: Cerebrospinal fluid; CT: Computed tomography; ERG: Electroretinogram; FFA: Fundus fluorescein angiography; ICA: Internal carotid artery; ICG: Indocyanine green angiography; MRA: Magnetic resonance angiography; MRI: Magnetic resonance imaging; NVD: Neovascularization of the disc; PCR: Polymerase chain reaction; TPHA: Treponema pallidum hemagglutination; VDRL: Venereal disease research laboratory; VEGF: Vascular endothelial growth factor
\end{abstract}

\section{Acknowledgements}

We thank Angela Morben, DVM, ELS, from Edanz Group

(www.edanzediting.com/ac), for editing a draft of this manuscript.

\section{Availability of data and materials}

Data for this case report were collected by a chart review of the patient's electronic medical record, which is not publicly available because of privacy considerations.

\section{Authors' contributions}

Study conception: PJ, KV, AP, and TT. Study design: KV, AP, and TT. Data acquisition: PJ and KV. Data analysis: KV, CD, PJ, PN, JW, PC, PP, and TS. Data interpretation: KV, CD, PJ, PN, JW, PC, PP, and TS. Drafting of the paper: KV, $A P, C D, P J, P N, J W, P C, P P, T$, and TS. Critical revision of the paper: KV. Final approval for submission: KV, AP, CD, PJ, PN, JW, PC, PP, TT, and TS.

\section{Ethics approval and consent to participate}

This study was approved by the Institutional Review Board of the Faculty of Medicine Ramathibodi Hospital and based on the Declaration of Helsinki.

The patient provided written informed consent prior to entering the study.

\section{Consent for publication}

Written informed consent was obtained from the patient for publication of this case report and any accompanying images. A copy of the consent form is available for review by the Editor of this journal.

\section{Competing interests}

The authors do not have any competing interests in the publication of this case report.

\section{Publisher's Note}

Springer Nature remains neutral with regard to jurisdictional claims in published maps and institutional affiliations.

\section{Author details}

'Department of Medicine, Faculty of Medicine Ramathibodi Hospital, Mahidol University, 270 Rama VI Road, Bangkok 10400, Thailand. 2Department of Pathology, Faculty of Medicine Ramathibodi Hospital, Mahidol University, 270 Rama VI Road, Bangkok 10400, Thailand. ${ }^{3}$ Department of Radiology, Faculty of Medicine Ramathibodi Hospital, Mahidol University, 270 Rama VI Road, Bangkok 10400, Thailand.

${ }^{4}$ Department of Ophthalmology, Faculty of Medicine Ramathibodi Hospital, Mahidol University, 270 Rama VI Road, Bangkok 10400, Thailand.

Received: 3 February 2018 Accepted: 3 May 2018

Published online: 08 May 2018

\section{References}

1. Dispenzieri A. POEMS syndrome: update on diagnosis, risk-stratification, and management. Am J Hematol. 2015;90:951-62.

2. Kaushik M, Pulido JS, Abreu R, Amselem L, Dispenzieri A. Ocular findings in patients with polyneuropathy, organomegaly, endocrinopathy, monoclonal gammopathy, and skin changes syndrome. Ophthalmology. 2011;118:778-82.

3. Zhang X, Cai QQ, Huang XF, Cao XX, Cai H, Zhou DB, Dai RP, Li J. Ocular manifestations and treatment outcomes in Chinese patients with poems syndrome. Retina. 2017:37:1784-91.

4. Imai H, Kusuhara S, Nakanishi Y, Escaño MF, Yamamoto H, Tsukahara Y, Negi A. A case of POEMS syndrome with cystoid macular edema. Am J Ophthalmol. 2005;139:563-6.

5. Prost MG, Gilhuis HJ, Brouwer RE, Gawda P. Local treatment with triamcinolone acetonide and bevacizumab for ocular symptoms in a patient with POEMS syndrome. Case Rep Ophthalmol. 2014;5:416-22.

6. Chong DY, Comer GM, Trobe JD. Optic disc edema, cystoid macular edema, and elevated vascular endothelial growth factor in a patient with POEMS syndrome. J Neuroophthalmol. 2007;27:180-3.

7. Ecsedy M, Schneider M, Nemes J, Nemeth J, Recsan Z. Optical coherence tomography features of POEMS syndrome and Castleman diseaseassociated papillopathy. Ocul Immunol Inflamm. 2014;22:454-60.

8. Kimura S, Morizane Y, Hosogi M, Hosokawa M, Shiode Y, Kawata T, Kondo E, Shiraga F. POEMS syndrome in a 20-year-old patient diagnosed following a complaint of reduced visual acuity. Acta Med Okayama. 2014;68:379-83.

9. Witoonpanich R, Phankhian S, Jootar S, Poonyathalang A, Worapongpaiboon S, Phudhichareonrat S, Chanplakorn N. POEMS syndrome with venous sinus thrombosis and visual failure: a case report. J Med Assoc Thail. 2005;88:690-4.

10. Bourdette DN, Rosenberg NL. Infiltrative orbitopathy, optic disk edema, and POEMS. Neurology. 1984;34:532-3.

11. Arnold PD, Kinyoun JL, Guzak SV. Poems syndrome: an unusual cause of neovascularization. Retina. 1999;19:166-8.

12. Diduszyn JM, Quillen DA, Cantore WA, Gardner TW. Optic disk drusen, peripapillary choroidal neovascularization, and POEMS syndrome. Am J Ophthalmol. 2002 Feb 28;133(2):275-6.

13. Rahimy E, Sarraf D. Paraneoplastic and non-paraneoplastic retinopathy and optic neuropathy: evaluation and management. Surv Ophthalmol. 2013 Oct 31;58(5):430-58

14. Dupont SA, Dispenzieri A, Mauermann ML, Rabinstein AA, Brown RD. Cerebral infarction in POEMS syndrome incidence, risk factors, and imaging characteristics. Neurology. 2009;73:1308-12.

15. Kang K, Chu K, Kim DE, Jeong SW, Lee JW, Roh JK. POEMS syndrome associated with ischemic stroke. Arch Neurol. 2003;60:745-9. 
16. Erro ME, Lacruz F, Aymerich N, Ayuso T, Soriano G, Gállego J, Villanueva JA. Acute carotid obliteration: a new vascular manifestation in POEMS syndrome. Eur J Neurol. 2003;10:383-4.

17. Garcia T, Dafer R, Hocker S, Schneck M, Barton K, Biller J. Recurrent strokes in two patients with POEMS syndrome and Castleman's disease. I Stroke Cerebrovasc Dis. 2007;16:278-84.

18. Fu FW, Rao J, Zheng YY, Wang HL, Yang JG, Zheng GQ. Ischemic stroke in patients with POEMS syndrome: a case report and comprehensive analysis of literature. Oncotarget. 2017;8:89406

19. Briani C, Fedrigo M, Manara R, Castellani C, Zambello R, Citton V, Campagnolo M, Dalla Torre C, Lucchetta M, Orvieto E, Rotilio A. Pachymeningeal involvement in POEMS syndrome: MRI and histopathological study. J Neurol Neurosurg Psychiatry. 2012;83:33-7.

Ready to submit your research? Choose BMC and benefit from:

- fast, convenient online submission

- thorough peer review by experienced researchers in your field

- rapid publication on acceptance

- support for research data, including large and complex data types

- gold Open Access which fosters wider collaboration and increased citations

- maximum visibility for your research: over $100 \mathrm{M}$ website views per year

At BMC, research is always in progress.

Learn more biomedcentral.com/submissions 